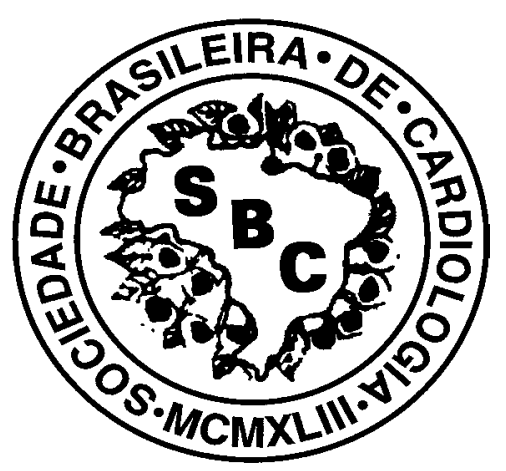

Normatização dos Equipamentos e Técnicas de Exame para Realização de Exames Ecocardiográficos 


\title{
Normatização dos Equipamentos e Técnicas de Exame para Realização de Exames Ecocardiográficos
}

\section{Editor}

Carlos Eduardo Suaide Silva

\author{
Membros \\ Rogério Tasca \\ Luiz Henrique Weitzel \\ Valdir Ambrósio Moisés \\ Luiz Darcy Cortez Ferreira \\ Glaucia Maria Penha Tavares \\ José Luiz Barros Pena \\ Juarez Ortiz \\ Caio C. J. Medeiros \\ Claudia Gianini Monaco \\ Luciana Braz Peixoto
}

\section{Coordenação}

Jorge Ilha Guimarães 
Arq Bras Cardiol

volume 82, (suplemento II), 2004

\section{I - Nomenclatura}

Ficam definidas as denominações ecocardiograma transtorácico, ecocardiograma transesofágico e ecocardiograma fetal para os exames realizados pelas vias respectivas (transtorácica, transesofágica e transabdominal ou transvaginal, para estudo do coração fetal).

Qualquer que seja a via do exame, é sempre necessária a realização integrada dos estudos em modo bidimensional + Doppler pulsátil + Doppler contínuo + mapeamento de fluxo em cores. Exames transtorácicos devem também incluir, obrigatoriamente, o estudo em modo $\mathrm{M}$.

Exames ecocardiográficos com realização parcial destas modalidades (por exemplo, somente modo M+ bidimensional + Doppler contínuo) são incompletos, fragmentam a informação e podem não fornecer dados indispensáveis ao manuseio clínico e terapêutico dos pacientes.

\section{II - Instrumentação básica}

1. Equipamentos ecocardiográficos - Os equipamentos de ultra-som utilizados para fins diagnósticos, na área de cardiologia, devem possuir programas de computador que permitam a realização de, no mínimo, os itens a seguir: a) estudo em modo $\mathrm{M}$; b) estudo em modo bidimensional; c) estudo com Doppler pulsátil e contínuo; d) estudo com mapeamento de fluxo em cores.

Para tal, torna-se necessário: a) monitor com dimensões e qualidade suficientes para interpretar todas as modalidades citadas. No monitor, deve constar o nome do laboratório de ecocardiografia, o nome do paciente, a data e a hora do exame, além do traçado eletrocardiográfico; b) capacidade do programa de, quando estiver utilizando uma modalidade derivada de outra (por exemplo, modo Mou Doppler), mostrar, simultaneamente, a imagem do estudo bidimensional (estática ou dinâmica), geradora dos modos $\mathrm{M}$ ou Doppler; c) escalas de medidas, que sempre devem aparecer em todas as modalidades; d) programa de computador capaz de realizar medidas de distância entre dois pontos, áreas, velocidades de fluxo sangüíneo, intervalos de tempo e gradientes médio e máximo nos estudos com Doppler; e) ao menos, dois transdutores setoriais, um de baixa freqüência (2 a 2,5 MHz) e um de alta freqüência (superior a 3,5 $\mathrm{MHz}$ ), ou um transdutor multifreqüencial que englobe essas faixas de freqüência; f) em caso de ecocardiograma transesofágico, pelo menos, um transdutor monoplanar, biplanar ou multiplanar (preferido), com freqüência de $5 \mathrm{MHz}$ ou mais alta. A sonda deve ser flexível e com dispositivos de controle de movimentos laterais e ântero-posteriores da extremidade distal onde está o transdutor. Toda sonda deve ter uma escala na superfície para orientação do operador quanto à distância de determinada estrutura ou anormalidade em relação à arcada dentária; g) em caso de ecocardiograma fetal, ao menos, um transdutor setorial de $5 \mathrm{MHz}$ (de preferência um transdutor convexo).
Diretriz para Normatização dos Equipamentos e Técnicas de Exame para Realização de Exames Ecocardiográficos

\section{III - Técnica de exame}

\section{Ecocardiograma transtorácico}

1.1 - Localização do transdutor (fig. 1) - a) janela paraesternal: região localizada entre o $2^{\circ} \mathrm{e} 4^{\circ}$ espaços intercostais esquerdos, junto à borda esternal; b) janela apical: região localizada junto ao ictus cordis; c) janela subcostal: região localizada na região epigástrica, junto ao apêndice xifóide; d) janela supraesternal: região localizada na fossa supraesternal; e)janela paraesternal direita: região localizada entre o $2^{\circ} \mathrm{e} \mathrm{o} 4^{\circ}$ espaço intercostal direito, junto à borda esternal; f)janela apical direita: região localizada junto ao ictus cordis, nos raros casos quando este pode ser palpado no lado direito do tórax.

Nota: Quando empregados os termos janela paraesternal e apical, fica subtendido tratar-se de localização esquerda. Os termos paraesternal e apical direitos serão empregados quando utilizadas as respectivas janelas.

1.2 - Planos de corte transtorácicos (fig. 2) - Os pla-

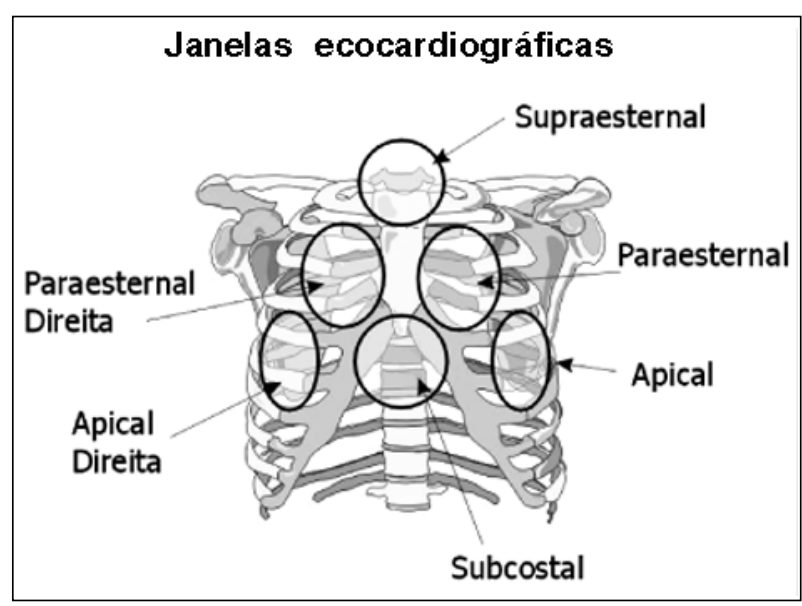

Fig. 1 - Janelas ecocardiográficas.

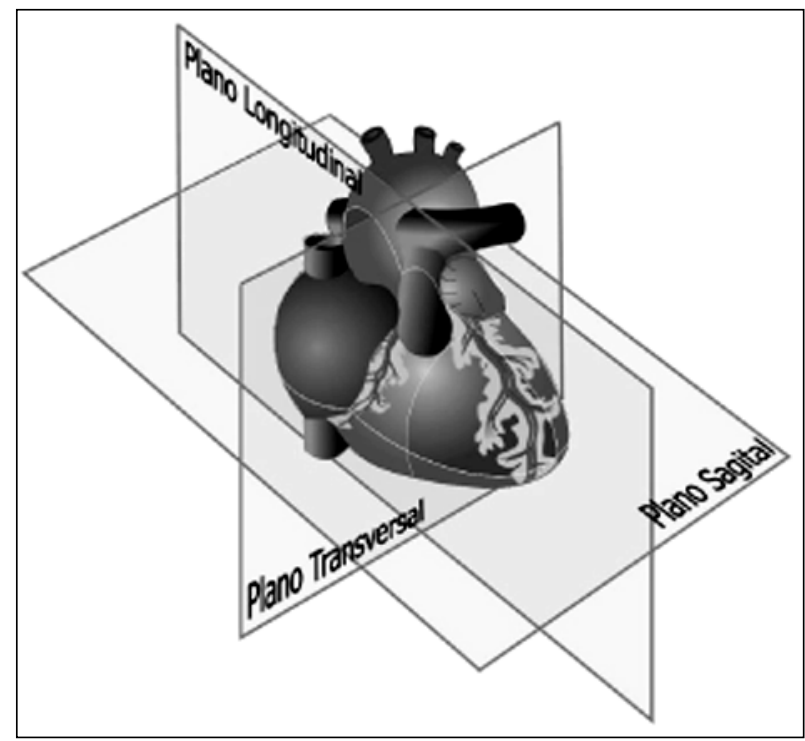

Fig. 2 - Planos ecocardiográficos. 
nos de cortes recomendados na ecocardiografia bidimensional transtorácica são baseados nas incidências em que o feixe do ultra-som incide no coração: a) plano longitudinal: plano de corte perpendicular à parede torácica e paralelo ao eixo longo do coração, numa linha imaginária que, geralmente, une o ombro direito ao flanco esquerdo no paciente normolíneo; b) plano transversal: plano de corte perpendicular à parede torácica e ao plano longitudinal; c) plano sagital: plano de corte aproximadamente paralelo à parede torácica e perpendicular aos planos longitudinal e transversal (correspondente ao corte apical de quatro câmaras).

Nota: Na janela supraesternal, são utilizados os planos longitudinal e transversal, que levam em consideração os respectivos eixos da aorta torácica.

1.3 - Nomenclatura dos cortes - A nomenclatura dos diversos cortes do cocardiograma bidimensional transtorácico deve incluir a localização do transdutor, seguida do plano de corte, como, por exemplo, corte paraesternal longitudinal; corte apical de 4 câmaras; corte paraesternal direito longitudinal; corte supraesternal transversal.

1.4 - Orientação da imagem - Índex: recomenda-se que todo aparelho de ecocardiografia bidimensional tenha uma marcação como índex, o qual deve indicar a borda do plano de imagem à direita do monitor(fig. 3). Durante a realização do exame ecocardiográfico, o transdutor deve estar localizado de tal forma que o índex esteja localizado em direção à cabeça ou ao lado esquerdo do paciente. As imagens serão apresentadas na tela do monitor com o ângulo do setor na sua extremidade superior. Opcionalmente, nas janelas apical e subcostal, as imagens podem ser dispostas anatomicamente, e o ângulo do setor aparecerá na extremidade inferior do monitor.

a) Cortes paraesternais (fig. 4) - São obtidos a partir da janela paraesternal, nos planos de cortes longitudinal e transversal. No corte paraesternal longitudinal, o ápex deverá aparecer à esquerda e a aorta à direita do monitor (A). No corte paraesternal ao nível dos músculos papilares, o septo anterior aparecerá na parte superior do monitor, o músculo papilar póstero-medial à esquerda e o ântero-lateral à direita, enquanto que a parede posterior do $\mathrm{VE}$, na região inferior do monitor (B). No corte paraesternal transversal ao nível da valva aórtica, o tronco pulmonar fica à direita e a valva tricúspide, à esquerda do monitor $(\mathrm{C})$.

b) Cortes apicais (fig. 5) - São obtidos a partir da janela apical. Os cortes apicais (de 2, 4 e 5 câmaras) devem mostrar o ápex cardíaco na parte superior do monitor (câmaras ventriculares) e a base na parte inferior (câmaras atriais), sendo que as câmaras esquerdas devem aparecer á direita (nos cortes de 4 e 5 câmaras) e a parede póstero-inferior, à esquerda (no apical de 2 câmaras). Opcionalmente, esses cortes podem ser apresentados com o ângulo setorial na região inferior do monitor (geralmente nos exames de crianças), bastando para tal pressionar o botão de inversão súperoinferior do aparelho de ecocardiografia.

c) Cortes supraesternais (fig. 6) - A) Longitudinal Como já relatado, este corte mostrará um plano longitudinal em relação à aorta torácica. Nesse corte, com o transdutor colocado na fúrcula esternal, o índex estará dirigido para o ombro esquerdo do paciente, obliquamente, numa linha imaginária entre a fúrcula e o 1/3 médio da clavícula esquerda. Nesta imagem, o arco aórtico estará localizado superiormente no monitor, enquanto que a aorta ascendente ficará a esquerda e o início da descendente, à direita; B) transversal - com uma rotação anti-horária de, aproximadamente, $90^{\circ}$, pode-se observar a aorta transversalmente, o tronco pulmonar à direita do monitor, a veia cava superior e a bifurcação da artéria pulmonar direita, à esquerda do monitor.

d) Cortes subcostais - $O$ corte de 4 câmaras pode ser
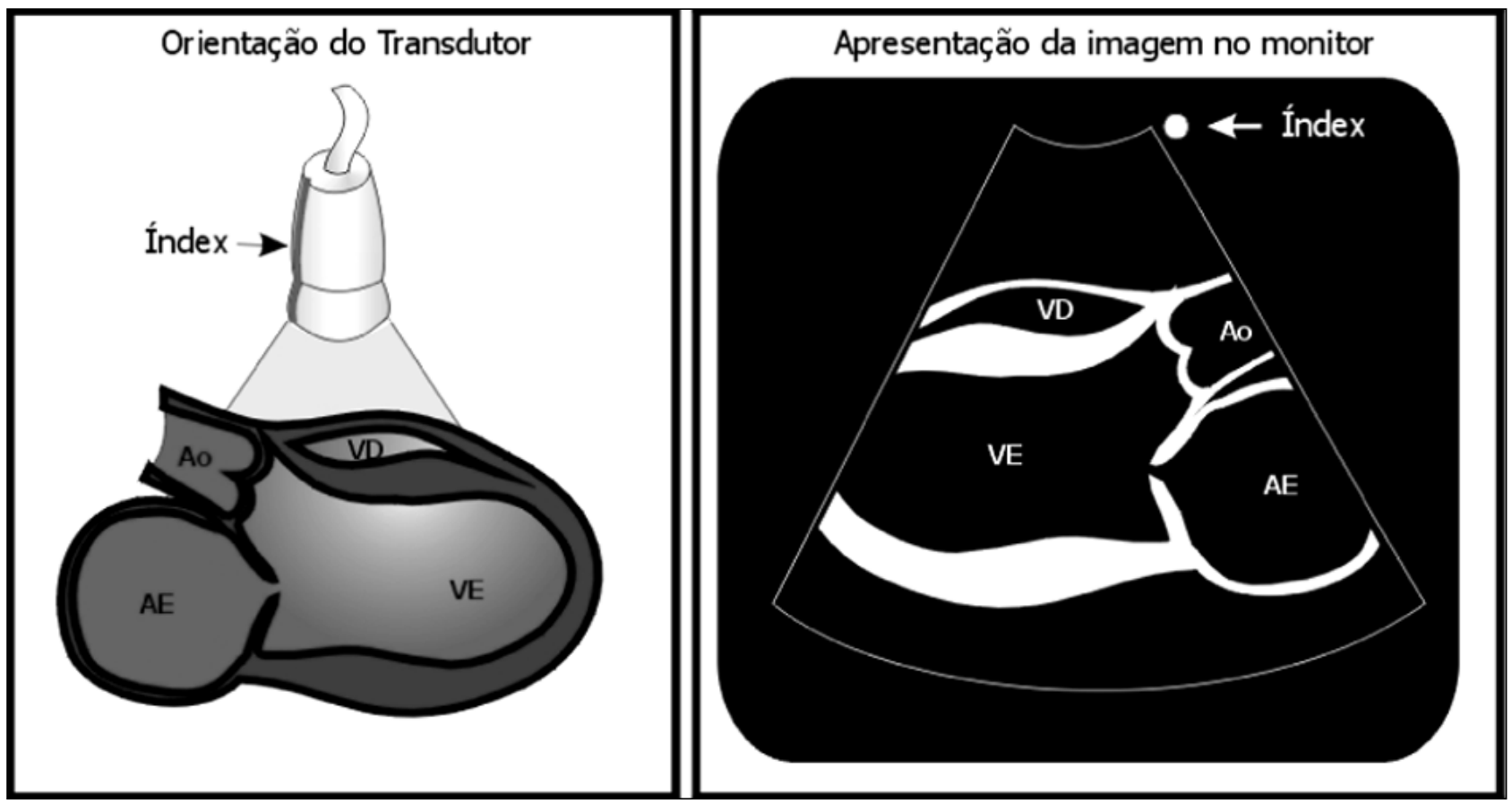

Fig. 3 - Orientação da imagem 
Arq Bras Cardiol

volume 82, (suplemento II), 2004

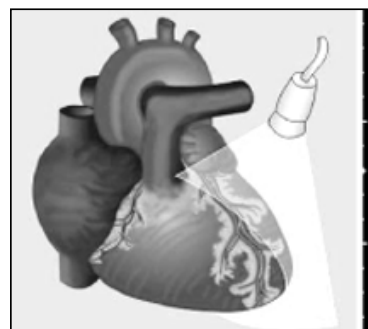

Paraesternal Longitudinal

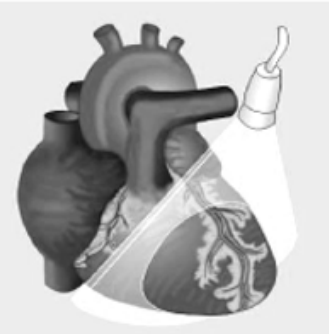

Paraesternal Transversal VE

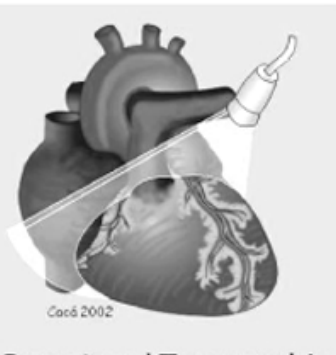

Paraesternal Transversal Ao
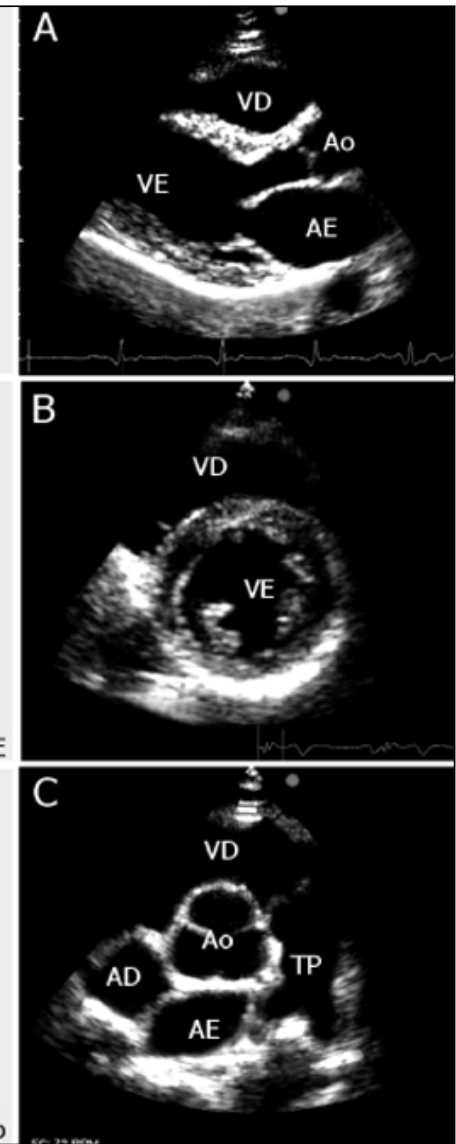

Fig. 4 - Cortes para esternais.

obtido com o transdutor na região subcostal, com o índex voltado para a esquerda do paciente Nesta imagem, o VD ficará na região superior do transdutor, o VE aparecerá na região inferior e à direita e o $\mathrm{AE}$, na região inferior e à esquerda. Da mesma forma, que no corte apical 4 câmaras, pode-se inverter a imagem, colocando o ângulo setorial na região inferior do monitor. Subcostal transversal é obtido com o índex voltado para a esquerda do paciente. Quando o corte é obtido ao nível dos músculos papilares, o VD aparecerá na parte superior do monitor, sendo que a parede posterior do VE será visibilizada na região inferior e à esquerda, o músculo papilar ântero-lateral, na região inferior e à direita e a parede anterior do VE, à direita do monitor. Mediante o corte dos átrios (fig. 7), são visibilizadas apenas as câmaras atriais, estando o átrio direito na parte superior e o esquerdo, na inferior. Esse é o corte de eleição para visibilização do septo interatrial.

1.5-Realização do exame -A) A realização do exame deve seguir técnica apropriada, conforme as normas do Departamento de Ecocardiografia da Sociedade Brasileira de Cardiologia.

Como em todo procedimento médico, deve-se respeitar o pudor dos pacientes, evitando exposições desnecessárias ou indevidamente protegidas de áreas do seu corpo.

Deve-se explicar ao paciente e/ou pais ou acompanhante, como é realizado o exame, diminuindo a ansiedade e a insegurança freqüentemente observadas.
Diretriz para Normatização dos Equipamentos e Técnicas de Exame para Realização de Exames Ecocardiográficos

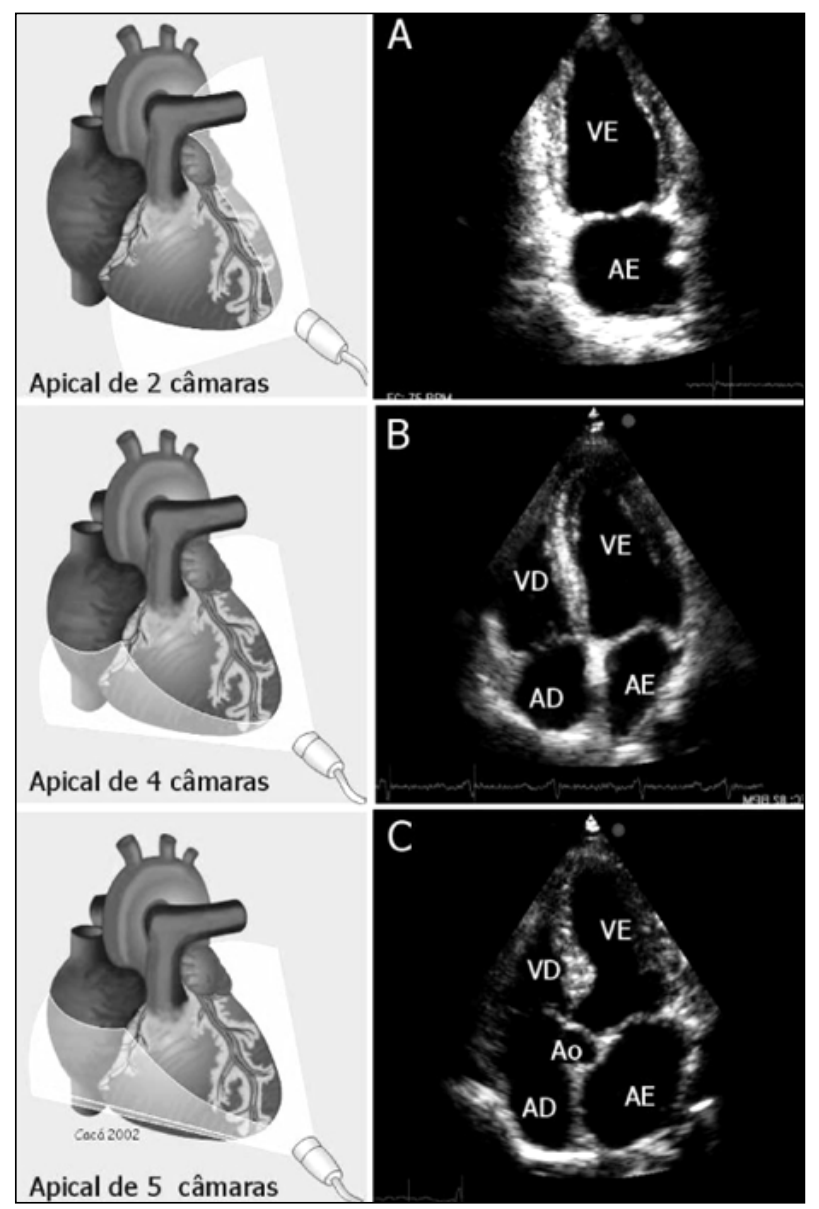

Fig. 5 - Cortes apicais.

É importante a realização de uma breve anamnese, procurando detectar o objetivo (ou objetivos) da solicitação do exame pelo médico assistente. A anamnese deve ser sempre complementada por ausculta cardíaca cuidadosa e, de acordo com critérios do examinador, também por aferição da pressão arterial.

No preparo do paciente e durante a realização do exame, o médico ecocardiografista poderá utilizar o auxílio de técnicos devidamente treinados, que atuarão sempre sob sua supervisão e responsabilidade.

O estudo ecocardiográfico deve examinar todas as câmaras e estruturas cardíacas e deve ser documentado.

B) A realização do exame deve incluir, ao menos: a) posicionamento adequado do paciente; b) escolha do transdutor ideal; c) otimização dos controles de ganho e do monitor do equipamento; d) realização dos estudos em modo M, bidimensional e Doppler (pulsátil, contínuo e colorido), de acordo com protocolos específicos da instituição; e) utilização apropriada das técnicas de Doppler (alinhamento do feixe) e medidas.

C) Elementos que contribuem para a boa qualidade do exame incluem, ao menos: a) definição do endocárdio; b) aquisição correta dos cortes ecocardiográficos; c) delineamento de detalhes da anatomia valvar; $\mathbf{d}$ ) medidas adequadas das dimensões do ventrículo esquerdo a partir dos 


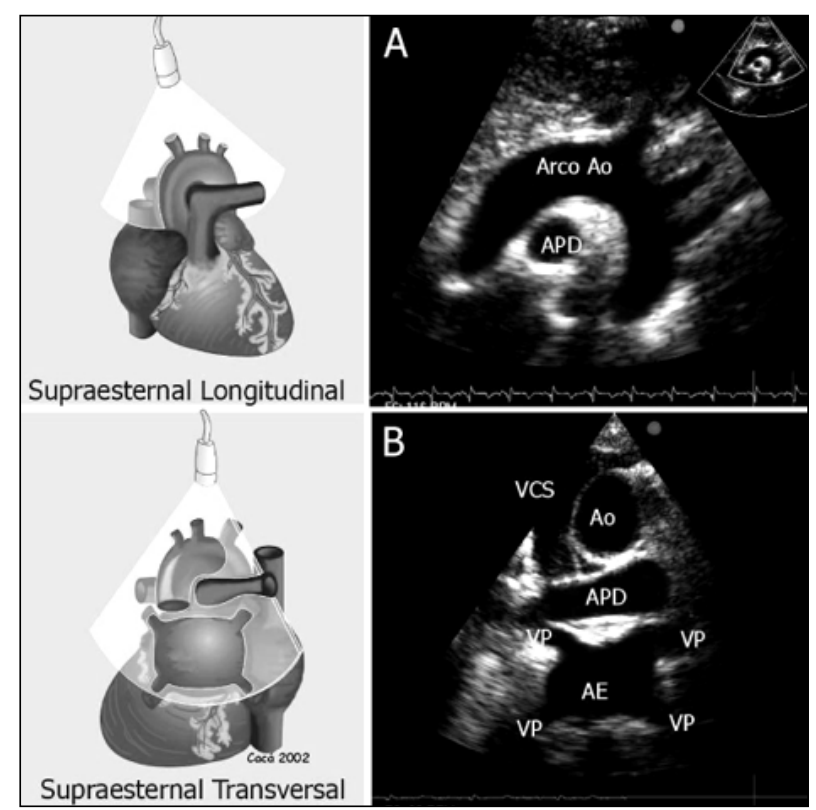

Fig. 6 - Cortes supra-esternais.

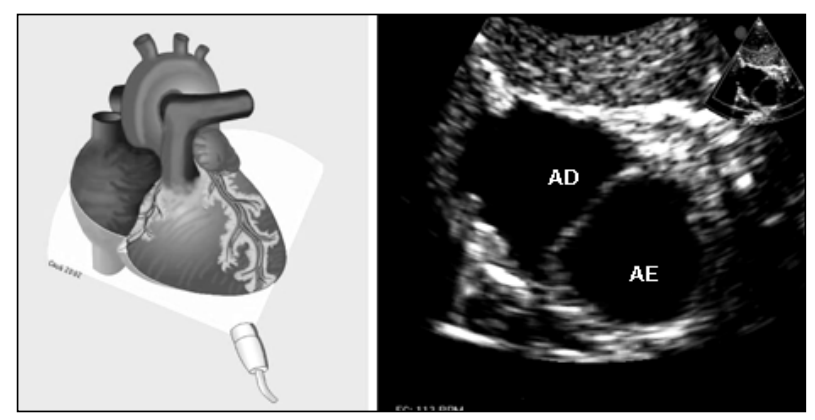

Fig. 7 - Corte subcostal.

cortes padronizados; e) aquisição e avaliação correta dos fluxos ao Doppler (o que deve incluir alinhamento paralelo do feixe com o fluxo); f) registro e documentação correta das curvas de fluxo ao Doppler (normais e alteradas) em múltiplos cortes; g) protocolo específico da instituição.

D) $\mathrm{O}$ exame ecocardiográfico transtorácico deve ser abrangente e incluir etapas padronizadas: D.1) etapas do exame: Um protocolo da instituição deve ser seguido com as orientações da seqüência e etapas do exame; a) estudo completo com os modos M e bidimensional-inclui cortes padronizados de vários planos com a visibilização de todas as estruturas cardíacas e de algumas extracardíacas, como ventrículo esquerdo, ventrículo direito, átrio esquerdo, átrio direito, valva aórtica, valva pulmonar, valva mitral, valva tricúspide, aorta proximal ascendente, aorta transversa, aorta proximal descendente, tronco da artéria pulmonar, artéria pulmonar esquerda, artéria pulmonar direita, veia cava inferior, veia hepática e pericárdio; b) estudo completo com Doppler-inclui o uso do Doppler pulsátil e contínuo e do mapeamento de fluxo em cores para a avaliação dos fluxos normais e anormais intracardíacos, abrangendo as valvas, grandes vasos e septos interatrial e interventricular. D.2) o exame completo deve in- cluir (a não ser que seja tecnicamente impossível), ao menos: a) os seguintes cortes ao estudo bidimensional; a1) paraesternal longitudinal; a2) paraesternal transversal (ao nível das valvas aórtica e mitral, e dos músculos papilares); a3) vias de entrada e de saída do ventrículo direito; a4) apical de quatro câmaras; a5) apical de duas câmaras; a6) apical de cinco câmaras; a7) apical longitudinal; a8) subcostal de quatro câmaras; a9) subcostal transversal (quando indicado); a10) subcostal da veia cava inferior e veia hepática; a11) supraesternal. b) as seguintes medidas ao estudo com modo Mou bidimensional: b1) diâmetro telediastólico interno do ventrículo esquerdo; b2) espessura telediastólica da parede póstero-inferior do ventrículo esquerdo; b3) espessura telediastólica do septo interventricular; b4) diâmetro telessistólico do átrio esquerdo; b5) diâmetro telediastólico da raiz da aorta. c) as seguintes medidas ao estudo com Doppler: c1) o fluxo anterógrado das quatro valvas cardíacas, assim como os fluxos retrógrados (se existentes), visibilizados ao mapeamento de fluxo em cores em, pelo menos, dois cortes ecocardiográficos; c2) na estenose aórtica, o fluxo de maior velocidade deve ser avaliado em múltiplos cortes (apical, supraesternal, paraesternal direito, etc); c3) estimativa da pressão sistólica pulmonar através do refluxo tricúspide, quando presente; c4) deve-se fazer uma varredura dos septos interatrial e interventricular com o mapeamento de fluxo em cores para detecção de defeitos, quantificação de seus diâmetros e dos gradientes intercavitários; c5) estimativa da velocidade do fluxo da via de saída do ventrículo esquerdo.

\section{Ecocardiograma transesofágico}

2.1 - Nome da técnica - Fica definido o nome ecocardiograma transesofágico para o exame ultra-sonográfico do coração e grandes vasos pela via transesofágica. Esta técnica compreende a intubação do esôfago com uma sonda semelhante à utilizada para endoscopia digestiva alta, com um ou mais transdutores na extremidade.

2.2 - O exame transesofágico - Esse exame pode ser realizado em pacientes ambulatoriais ou internados, em laboratório de ecocardiografia, enfermarias ou unidades de terapia intensiva, durante realização de procedimentos diagnósticos ou terapêuticos na sala de hemodinâmica e durante cirurgia cardíaca ou não cardíaca no centro cirúrgico.

2.2.1 - Laboratório de ecocardiografia - Para a realização do ecocardiograma transesofágico, o laboratório deve ser equipado com instrumentos e medicamentos de ressuscitação cardiorrespiratória, laringoscópio e sondas de intubação traqueal, cardioversor elétrico e monitor de saturação de oxigênio (oxímetro de pulso). Além disso, deve haver água corrente e reservatório com substância antiséptica para limpeza e desinfecção do transdutor.

2.2.2 - Realização do exame no laboratório de ecocardiografia - $\mathrm{O}$ exame transesofágico deve ser precedido pela realização do exame transtorácico, que pode, entretanto, ser dispensado se já tiver sido realizado recentemente e não tenham ocorrido mudanças no quadro clínico, ou se as condições clínicas do paciente não permitirem.

Para todos os tipos de ecocardiograma transesofágico, recomenda-se jejum absoluto de, pelo menos, 5 horas. 
Arq Bras Cardiol

volume 82, (suplemento II), 2004
Diretriz para Normatização dos Equipamentos e Técnicas de Exame para Realização de Exames Ecocardiográficos
Deve-se explicar a rotina do exame ao paciente e convencê-lo da necessidade de cooperação, assim como questioná-lo sobre possíveis doenças esofágicas que dificultam ou impeçam a intubação, para evitar complicações.

Antes da intubação do esôfago, deve-se retirar todo tipo de prótese dentária removível e utilizar protetor de mordeduras

Recomenda-se analgesia da orofaringe com anestésico tópico, punção de veia periférica e, se necessário, é preferível uso de sedativos por via endovenosa.

Durante todo o exame, é recomendado que o paciente permaneça em decúbito lateral (esquerdo ou direito). Após o final do exame e a retirada da sonda, deve ser mantido nessa posição até estar totalmente consciente. Após a retirada da punção venosa, o paciente deve ficar em repouso no laboratório por, pelo menos, $30 \mathrm{~min}$, para observação.

2.2.3 - Nas unidades de terapia intensiva, o procedimento é semelhante. Devem ser avaliadas as condições clínicas do paciente e, com a equipe clínica, definida a melhor estratégia.

2.2.4 - Na sala de hemodinâmica, o exame é realizado, em geral, com o paciente em decúbito dorsal, sendo recomendada sedação para conforto do paciente e do médico.

2.2.5 - No centro cirúrgico, o exame sempre é realizado com o paciente na posição adequada para o procedimento cirúrgico (em geral, decúbito dorsal horizontal), e a intubação do esôfago é realizada após indução anestésica.

2.3 - Localização do transdutor - Após a intubação do esôfago, a posição da extremidade do transdutor é orientada a partir da distância da arcada dentária superior. Os planos são: esôfago superior (20 a $25 \mathrm{~cm}$ da arcada dentária), esôfago médio ( 30 a $40 \mathrm{~cm}$ da arcada dentária), transgástrico ( 40 a $45 \mathrm{~cm}$ da arcada dentária) e transgástrico profundo (45 a $50 \mathrm{~cm}$ da arcada dentária) (fig. 8, tab. I). Em cada um dos planos, vários cortes e imagens podem ser obtidos. Com o

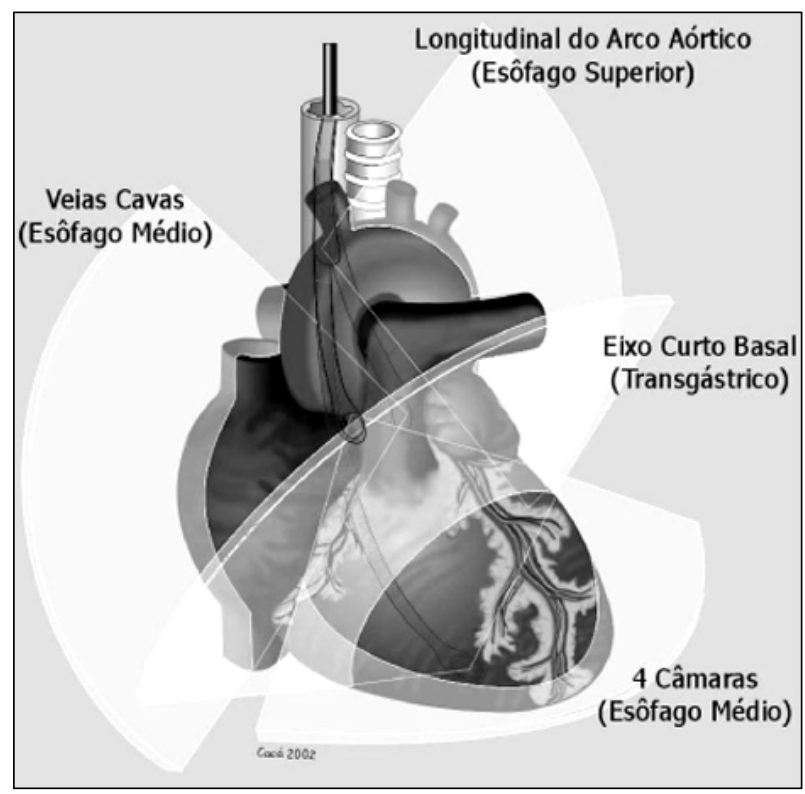

Fig. 8 - Diversos níveis de cortes transesofágicos. transdutor multiplanar, pode-se rodar o eixo de zero a 180 graus. Independentemente do tipo de transdutor, ajustes manuais devem ser feitos para que as imagens desejadas sejam obtidas (fig. 9).

Sempre que possível, os segmentos ascendente, transverso e descendente da aorta torácica devem ser analisados.

2.4 - Orientação da imagem - As imagens serão apresentadas na tela do monitor com o ângulo do setor na sua extremidade superior, com o índex à esquerda da tela. Nessa situação, no plano do esôfago médio, o átrio esquerdo estará sempre na parte superior e a ponta do ventrículo esquerdo orientada para baixo e à esquerda da tela (direita do operador).

2.5-Realização do exame -A) deve ser sempre realizado por médico treinado, que deve seguir a técnica apropriada, conforme as normas do Departamento de Ecocardiografia da Sociedade Brasileira de Cardiologia. Deve-se explicar ao paciente, e/ou pais ou acompanhante, como é realizado o exame. O estudo ecocardiográfico deve examinar todas as câmaras e estruturas cardíacas e ser sempre documentado. B) a realização do exame deve incluir, ao menos: a) posicionamento adequado do paciente; b) otimização dos controles de ganho e do monitor do equipamento; c) obtenção de imagens em modo M, bidimensional e Doppler (pulsátil, contínuo e de mapeamento de fluxo em cores), de acordo com protocolos específicos da instituição. C) Elementos que contribuem para boa qualidade do exame incluem, ao menos: a) definição do endocárdio; b) aquisição correta dos cortes ecocardiográficos; c) delineamento de detalhes da anatomia; d) medidas adequadas das dimensões do ventrículo esquerdo a partir dos cortes padronizados (podem ser obtidas pelo exame transtorácico); e) aquisição e avaliação correta dos fluxos ao Doppler (o que deve incluir alinhamento paralelo do feixe com o fluxo); f) registro e documentação correta das curvas de fluxo ao Doppler (normais e alteradas) em múltiplos cortes; g) protocolo específico da instituição. O exame ecocardiográfico transesofágico deve ser abrangente, com obtenção de imagens em todos os planos possíveis e incluir etapas padronizadas, como identificação e registro da anatomia e integridade do septo atrial, da anatomia e função do apêndice atrial esquerdo, análise de veias pulmonares, estrutura e função das valvas, particularmente da mitral e análise de todos os segmentos da aorta torácica. Para avaliação mais precisa de possível forame oval patente, pode-se usar injeção por veia periférica de solução salina, manualmente agitada, e manobras respiratórias.

2.6-Exames em crianças - Para a realização do exame em crianças pequenas, recomenda-se uso de transdutores e sondas apropriadas, de diâmetro e comprimento menores. $\mathrm{O}$ exame é, em geral, realizado em salas de hemodinâmica, ambiente de terapia intensiva ou no centro cirúrgico, preferencialmente sob assistência de um anestesista. Em crianças maiores ou adolescentes, após avaliação, pode ser tentado o exame em sala de laboratório de ecocardiografia, com o transdutor habitualmente utilizado em adultos, tomando-se os devidos cuidados com a sedação, ventilação e possíveis traumas ou complicações. 


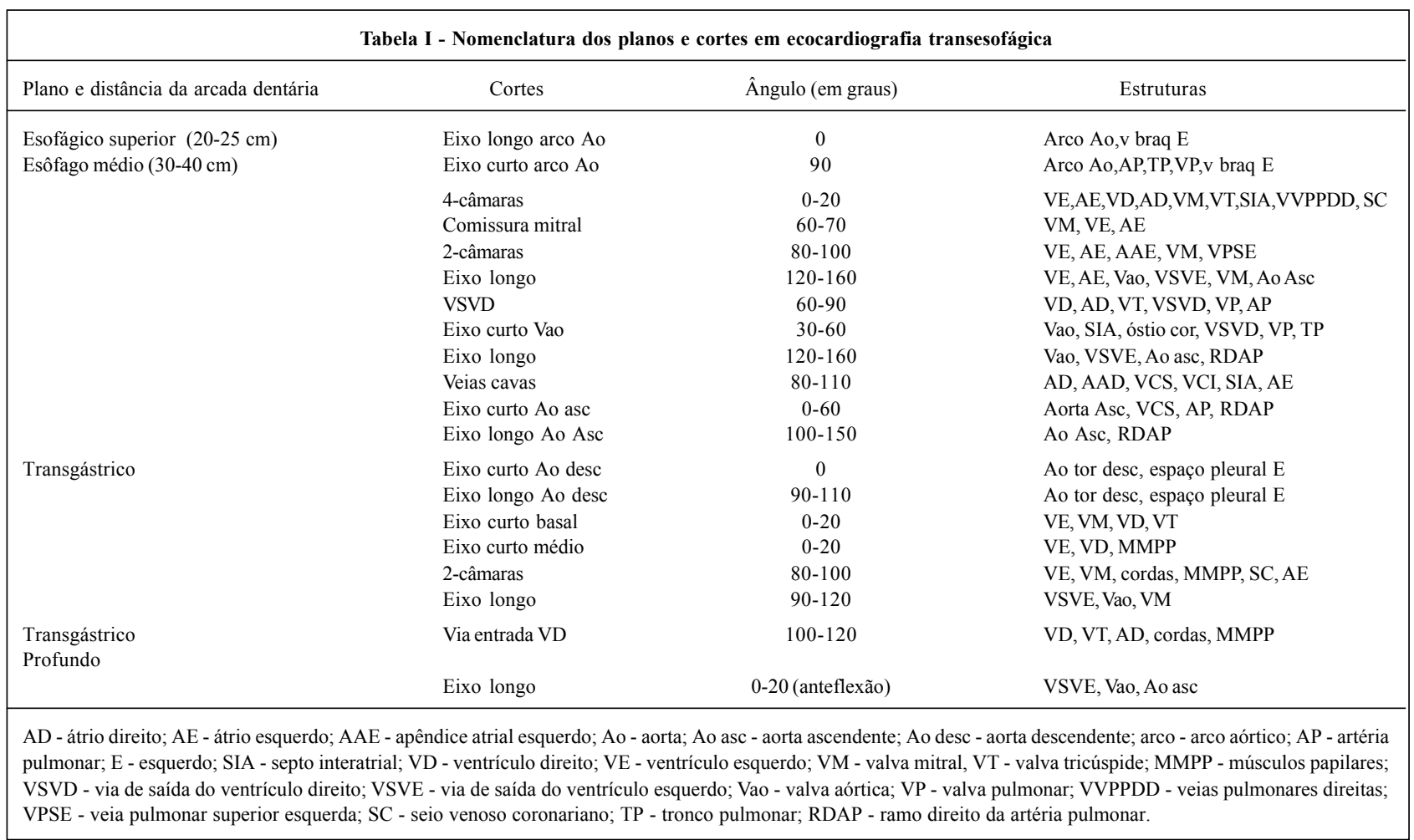

\section{Cortes Transesofágicos}

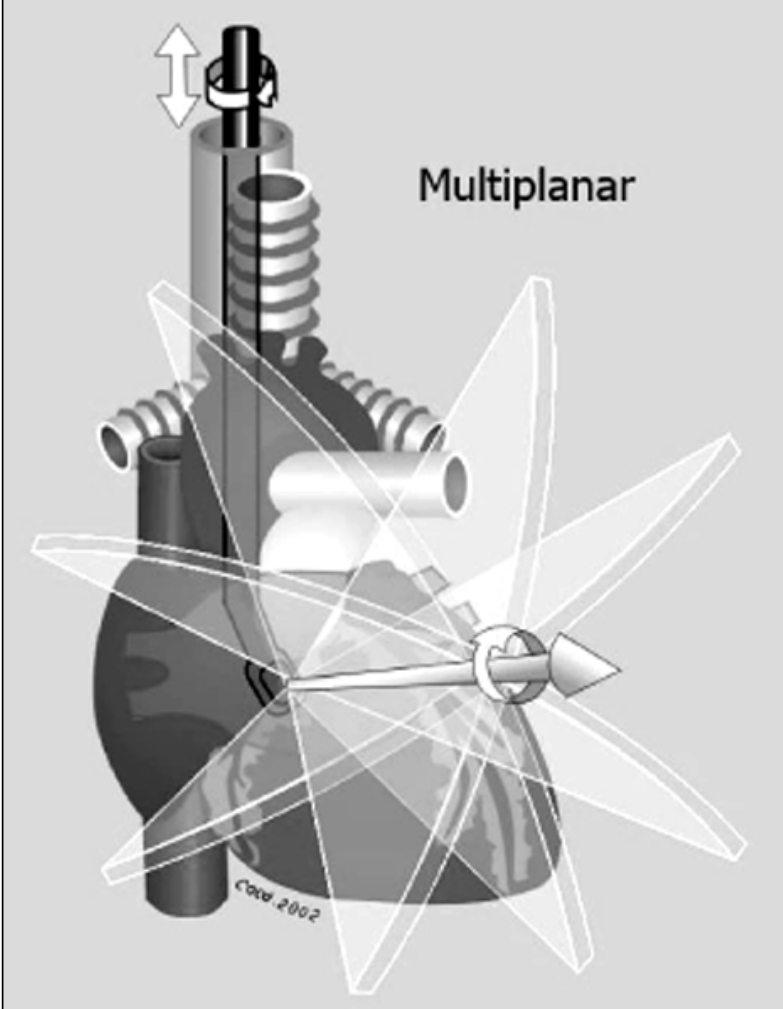

Fig. 9 - Diversos ângulos de corte do ecocardiograma transesofágico multiplanar.

\section{Ecocardiograma Fetal}

3.1 - Nome da técnica - Fica definido o nome ecocardiograma fetal para o exame ultra-sonográfico realizado na gestante para a análise do coração e grandes vasos do(s) feto(s).

3.2 -Momento da realização do exame - Apesar de ser tecnicamente possível realizar o ecocardiograma fetal a partir da $8^{\mathrm{a}}$ ou $10^{\mathrm{a}}$ semana de gestação até o termo, a qualidade da imagem obtida sofre influência da idade gestacional, sendo menos adequada antes da $18^{\mathrm{a}} \mathrm{e}$ após a $34^{\mathrm{a}}$ semana.

Em situações excepcionais quando há indicação específica de realização precoce do exame (entre 12 e 18 semanas), pode ser empregada a técnica através da via transvaginal.

Apesar desta possibilidade, o Boletim Técnico para a Realização do Exame de Ultra-som Cardíaco Fetal do American Institute of Ultrasound in Medicine (AIUM) recomenda que o exame seja feito, idealmente, entre a $18^{\mathrm{a}}$ e a $22^{\mathrm{a}}$ semanas de gestação, quando a qualidade da imagem é melhor e propicia maior acuidade diagnóstica.

Mesmo com 18 semanas de gestação, o coração fetal tem dimensões muito pequenas e ainda pode sofrer alterações estruturais e funcionais na evolução da gravidez, especialmente na presença de malformações estruturais cardíacas. Desta forma, quando o primeiro exame é realizado atéa $22^{\mathrm{a}}$ semana e/ou considerado insuficiente para a definição diagnóstica, recomenda-se uma ou mais reavaliações seqüenciais.

3.3 - Transdutores - Para a obtenção de imagens de resolução adequada, utilizam-se, para a via transabdominal, 
Arq Bras Cardiol

volume 82, (suplemento II), 2004
Diretriz para Normatização dos Equipamentos e Técnicas de Exame para Realização de Exames Ecocardiográficos transdutores de freqüência alta ( $5 \mathrm{MHz}$ ou mais), setoriais ou convexos. Entretanto, fatores, como o biótipo materno, a quantidade de líquido amniótico, a interposição de estruturas extracardíacas que determinam sombra acústica sobre a imagem cardíaca devido à posição fetal ou à idade gestacional avançada, podem exigir a utilização de transdutores com freqüência mais baixa $(2,5$ a 3,5 MHz). Quando é empregada a técnica transvaginal, deve-se utilizar a sonda endovaginal de $9 \mathrm{mHz}$.

3.4 - Realização do exame - A) Deve ser sempre realizado por médico treinado, seguindo a técnica apropriada, de acordo com as normas do Departamento de Ecocardiografia da Sociedade Brasileira de Cardiologia. O exame deve ser explicado à gestante e ao acompanhante, antes do seu início, quanto à técnica, objetivos, limitações e previsão do tempo de duração. O estudo deve examinar todas estruturas cardíacas, analisando os aspectos anatômico, funcional e do ritmo cardíaco, e ser documentado.

B) Arealização do exame deve incluir, ao menos: a) posicionamento adequado e confortável da paciente; b) escolha do transdutor e ajuste da freqüência de uso ideal; c) otimização dos controles de ganho e do monitor do equipamento; d) obtenção de imagens em modo bidimensional, Modo M e Doppler (pulsátil e mapeamento de fluxo em cores rotineiramente; contínuo apenas se necessário, em algumas situações), de acordo com protocolos específicos da instituição; e) utilização apropriada das técnicas de Doppler (alinhamento do feixe) e medidas.

C) Elementos que contribuem para a boa qualidade do exame incluem, ao menos: a) idade gestacional, biótipo materno e posição fetal favoráveis, quantidade normal de liquido amniótico; b) definição do endocárdio; c) aquisição correta dos cortes ecocardiográficos; d) delineamento de detalhes da anatomia cardíaca; e) medidas adequadas das dimensões das câmaras e demais estruturas cardíacas ao modo bidimensional e/ou modo $\mathrm{M}$, a partir dos cortes padronizados; f) aquisição e avaliação correta dos fluxos ao Doppler (o que deve incluir alinhamento do feixe o mais paralelo possível ao fluxo); g) registro e documentação correta das curvas de fluxo ao Doppler (normais e alteradas) em múltiplos cortes; h) seguir protocolo específico da instituição.

D) O exame ecocardiográfico fetal deve ser abrangente e incluir as etapas padronizadas. D.1) etapas do exame: um protocolo da instituição deve ser obedecido, com as orientações da seqüência e etapas do exame, que devem ser seguidas, sempre que possível. a) Estudo completo com os modos bidimensional e modo $\mathrm{M}$ - inclui cortes padronizados de vários planos, com a visibilização de todas as estruturas cardíacas e de algumas extracardíacas, como átrios direito e esquerdo; ventrículos direito e esquerdo; valvas aórtica, pulmonar, mitral e tricúspide; aorta ascendente, arco aórtico e aorta descendente; tronco e ramos da artéria pulmonar; arco ductal; veias cavas superior e inferior; veias pulmonares; septo interatrial com o forame oval pérvio; septo interventricular; pericárdio; b) estudo completo com Dopplerinclui o uso do Doppler pulsátil e, eventualmente, contínuo e o mapeamento do fluxo em cores para a avaliação dos flu- xos normais e anormais nas valvas, grandes vasos, arcos aórtico e ductal, forame oval e septo interventricular. D.2-O exame completo deve incluir (a não ser que seja tecnicamente impossível), ao menos: a) os seguintes cortes, ao estudo bidimensional: A1 - quatro câmaras; A2 - via de saída do ventrículo esquerdo; A3 - via de saída do ventrículo direito; A4 - arco aórtico; A5 - arco ductal. b) as seguintes medidas ao estudo com modo $\mathrm{M}$ ou bidimensional: B1 - diâmetro diastólico interno do ventrículo esquerdo; B2 - espessura diastólica do septo interventricular; B3 - espessura diastólica da parede póstero-inferior do ventrículo esquerdo; B4 - diâmetro sistólico interno do ventrículo esquerdo; B5 - anel valvar aórtico na sístole; B6 - anel valvar pulmonar na sístole; B7 - anel valvar mitral na diástole; B8 - anel valvar tricúspide na diástole; B9 - aorta ascendente; B10 tronco da artéria pulmonar. c) As seguintes medidas ao estudo com Doppler: C1 - o fluxo anterógrado das quatro valvas cardíacas, assim como os fluxos retrógrados, se existentes, visibilizados ao mapeamento de fluxo em cores; C2 o fluxo através do forame oval pérvio; C3 - o fluxo através dos arcos aórtico e ductal.

3.4 - Orientação da imagem - Convencionalmente, inicia-se o exame com o índex do transdutor localizado à direita da gestante, com as imagens apresentadas na tela do monitor com o ângulo do setor na sua extremidade superior.

Devido ao movimento e à variabilidade do posicionamento fetal, é necessário que o examinador defina a situação, posição e apresentação do feto antes de iniciar a avaliação específica do coração. Após estabelecidos com exatidão esses parâmetros, é possível analisar adequadamente as relações espaciais das estruturas do sistema cardiovascular entre si e em relação aos demais órgãos fetais adjacentes. Fica a critério do examinador, após definir a localização fetal, manter a orientação convencional do transdutor ou adequá-la para obter as imagens do coração fetal em posição anatômica, facilitando sua interpretação.

3.5 - Cortes ecocardiográficos - Definição do situs cardíaco: é inferido através da análise da posição da aorta e da veia cava inferior no abdome fetal, em cortes transversal e longitudinal. Pode também ser feita através da análise dos apêndices atriais no corte do eixo curto transversal cardíaco, nem sempre fácil de ser obtida no feto. Quatro câmaras: obtido a partir de corte transversal da metade inferior do tórax fetal, ajustando-se a imagem de tal forma que o estômago ou outros órgãos abdominais não sejam visíveis concomitantemente. Na situação de normalidade, o coração deve ocupar, no máximo, um terço da área do tórax e ter o ápice apontado para a esquerda do feto. Os átrios e os ventrículos devem ter tamanhos semelhantes entre si. Pode ser avaliada a amplitude da abertura da membrana da fossa oval, que prolapsa para o átrio esquerdo, e a integridade do septo interventricular. Identifica-se a banda moderadora no ventrículo direito, devendo essa cavidade estar contralateral ao átrio esquerdo, já identificado pela presença da membrana da fossa oval. Pode também ser observado o plano de inserção das valvas atrioventriculares, com discreto deslocamento apical da valva tricúspide em relação à mitral. Via de saída do ventrículo esquerdo: pode ser analisada a par- 
tir da angulação anterior e rotação horária do corte de quatro câmaras, expondo a aorta, ou através da obtenção de um corte semelhante ao paraesternal eixo longo. Permite analisar a dimensão e a eventual presença de estruturas anômalas que obstruam a via de saída do ventrículo esquerdo, além de complementar a análise da integridade do septo interventricular. Via de saída do ventrículo direito: pode ser analisada a partir da angulação anterior do corte de quatro câmaras, expondo a artéria pulmonar que deve se direcionar para a esquerda, cruzando por sobre a aorta. Também pode ser vista em corte semelhante ao paraesternal eixo curto, em que se vê a valva aórtica centralmente em corte transversal, os dois átrios, a conexão do átrio com o ventrículo direito e deste com a artéria pulmonar, além de sua bifurcação. A partir desse corte, pode ser obtido o arco ductal, através do prolongamento da imagem do tronco e ramos pulmonares, em plano para-sagital oblíquo, com a coluna no limite inferior da imagem, em corte longitudinal. Arco aórtico: em plano de sagital oblíquo do feto, com a coluna em seu limite inferior vista em corte longitudinal, é possível ver a aorta ascendente, o arco aórtico com a emergência de suas artérias e a aorta descendente. Arco ductal: pode ser obtido a partir da angulação anterior do corte do arco aórtico, devido à superposição destas duas estruturas, ou a partir do eixo curto da via de saída do ventrículo direito. $\mathrm{O}$ arco ductal difere do arco aórtico pelas peculiaridades de determinar em sua ex- tremidade distal um ângulo quase reto com a aorta descendente, e de não apresentar emergência de outros vasos em seu trajeto.

3.6 - Análise do Doppler e mapeamento do fluxo em cores - Assim como nas outras modalidades da ecocardiografia, a avaliação morfológica ao modo bidimensional deve ser complementada com o estudo dos fluxos pela análise do Doppler e mapeamento em cores. Para a obtenção de curvas adequadas ao Doppler pulsátil, procura-se alinhar a amostra o máximo possível paralelamente ao fluxo a ser estudado, nos diversos cortes. A direção das curvas e o sentido dos fluxos variam com a posição fetal em relação ao transdutor, podendo, inclusive, se modificar durante o exame, de acordo com a movimentação fetal.

3.7 - Análise da função cardíaca - Deve ser feita a partir das medidas ao modo $\mathrm{M}$, em corte transversal dos ventrículos. Quando não for possível obter imagem adequada, deve ser feita a análise qualitativa.

3.8 - Análise do ritmo cardíaco - Deve ser feita a partir da análise do movimento mecânico da contração atrial e ventricular ao modo bidimensional e de medidas obtidas do traçado do modo M simples ou colorido, e/ou através das curvas de Doppler. É o melhor método para a análise do ritmo cardíaco fetal, considerando a dificuldade técnica e até mesmo impossibilidade de monitoração eletrocardiográfica do feto. 